wird daraufhin die CD-Rom „madatinfo 1.0“. Aufbauend auf einer Variablen-Vorauswahl, die mit Hilfe dieser Produkte getroffen werden kann, werden für die Nutzer individuelle ma-Datensätze nach Wunsch extrahiert.

Weitere Informationen sind in aktualisierter Form auf der Homepage http://www.wiso.unikoeln.de/medien zu finden. Das MLFZ sucht Kooperationspartner und -einrichtungen medienund sozialwissenschaftlicher Natur im gesamten deutschsprachigen Raum.

Ansprechpartner beim MLFZ: Jörg Hagenah, Universität zu Köln, Medienwissenschaftliches Lehr- und Forschungszentrum, Wirtschafts- und Sozialwissenschaftliche Fakultät, Lindenburger Allee 15, D-50931 Köln, Tel. 0221/470-3953; E-Mail: <hagenah@wiso.uni-koeln.de>.

\section{CALL FOR PROPOSALS}

Themenschwerpunkt im ALLBUS 2006: "Ausländer / Ethnische Gruppen / Minderheiten"

Die Allgemeine Bevölkerungsumfrage der Sozialwissenschaften (ALLBUS) ist ein Serviceangebo der „Gesellschaft Sozialwissenschaftlicher Infrastruktureinrichtungen" (GESIS) für Forschung und Lehre in den Sozialwissenschaften. Seit 1980 werden im ALLBUS alle zwei Jahre Indikatoren zu Einstellungen, Verhaltensweisen und Sozialstruktur der Bevölkerung in der Bundesrepublik Deutschland erhoben. Die Daten jeder Erhebung stehen unmittelbar im Anschluss an ihre Aufbereitung der sozialwissenschaftlichen Profession für Sekundäranalysen zur Verfügung.

Für die ALLBUS-Erhebung 2006 ist die Replikation des Themenschwerpunktes „Einstellungen gegenüber ethnischen Gruppen in Deutschland" aus dem ALLBUS 1996 vorgesehen. Mit dem ca. 30-minütigen Fragenprogramm zu diesem Thema wurden die Einstellungen der Deutschen gegenüber verschiedenen ethnischen Gruppen in ihren unterschiedlichen Dimensionen sowie mögliche Determinanten dieser Einstellungen erfaßt (vgl. zum Fragenprogramm des ALLBUS 1996 im Einzelnen und zu den zahlreichen Veröffentlichungen dazu: http://www.gesis.org/ Dauerbeobachtung/Allbus/service_guide.htm).

Mit diesem Aufruf möchten wir der Profession die Gelegenheit geben, an der Konzeption des Schwerpunktes „Ausländer/Ethnische Gruppen/Minderheiten" für den ALLBUS 2006 mitzuwirken. Willkommen sind sowohl Vorschläge für neue Einzelfragen und Fragemodule zu die- sem Themenkomplex als auch kritische Rückmeldungen und Modifikationsvorschläge zum Fragenprogramm von 1996. Die Vorschläge für neue Fragen sollten theoriegeleitet sein, es sollte die sozialwissenschaftliche Relevanz begründet und die Existenz einschlägiger, für allgemeine Bevölkerungsumfragen tauglicher Indikatoren belegt werden (gerne auch aus Studien in anderen Ländern). Neu zu entwickelnde Fragen sollten kurz skizziert werden. Sowohl neue Fragevorschläge als auch Stellungnahmen zum Fragenprogramm von 1996 sollten bis spätestens 31. Mai 2004 bei uns eingehen.

Über die Berücksichtigung der Vorschläge entscheidet der wissenschaftliche Beirat des ALLBUS. Alle Bewerber werden unverzüglich über den Ausgang des Verfahrens informiert. Die ausgewählten Vorschläge werden von der ALLBUSAbteilung bei ZUMA in Abstimmung mit den Autoren weiter ausgearbeitet, getestet und in ein feldfähiges Befragungsinstrument überführt. Die Entscheidung über Aufnahme und Formulierung von Fragen liegt bei der ALLBUS-Abteilung und dem wissenschaftlichen Beirat des ALLBUS. Bitte senden Sie Ihre Vorschläge bis spätestens 31 . Mai 2004 an: Martina Wasmer, Postfach 1221 55, D-68072 Mannheim, wasmer@zuma-mannheim. de, Tel. 0621/1246-273.

\section{CALL FOR PAPERS}

"The State of the Art of Qualitative Social Research in Europe"

Joint Workshop organized by the ESA Research Network Qualitative Methods,

the DGS Section Qualitative Methoden and the SGS Research Committee Interpretive Social Research

Qualitative methods have been a constitutive part of empirical social research from its earliest days. But for a long period of time qualitative methods have also been regarded as an artwork rather than a scientific way of doing sociology. In recent times however, these methods have become more and more accepted. For instance, the German Society of Sociology (DGS) just decided to include them into the curriculum for Empirical Social Research on equal terms with methods. Qualitative methods today provide major instruments for social research on the level of fundamental scientific questions, as well as in the applied social research. Ranging from the EU to 